\title{
Geração de Imagens PET Estáticas com [11 C]-(R)-PK11195: Definição do Intervalo Temporal
}

\author{
Generation of Static PET images with $\left[{ }^{11} \mathrm{C}\right]-(R)-\mathrm{PK} 11195$ : \\ Defining Time Interval
}

\author{
Phelipi Nunes Schuck ${ }^{1}$, Caroline Machado Dartora' ${ }^{1}$, Ana Maria Marques da Silva ${ }^{1}$ \\ ${ }^{1}$ PUCRS, Núcleo de Pesquisa em Imagens Médicas, Porto Alegre, Brasil
}

\begin{abstract}
Resumo
O radiotraçador $\left[{ }^{11} \mathrm{C}\right]-(R)-\mathrm{PK} 11195$ mostra afinidade com a microglia em imagens PET in vivo e vem sendo utilizado como indicador de doença inflamatória cerebral, como na esclerose múltipla (EM). Atualmente, não há consenso sobre o intervalo temporal adequado para gerar imagens PET estáticas com $\left[{ }^{11} \mathrm{C}\right]-(R)-\mathrm{PK} 11195$. O objetivo deste trabalho é determinar o intervalo temporal mais adequado para a geração de imagens PET cerebrais estáticas adquiridas com [ $\left.{ }^{11} \mathrm{C}\right]-(R)$-PK11195 para a quantificação. Para este estudo foram utilizadas imagens de 10 pacientes com EM remitente-recorrente e 5 indivíduos saudáveis. Foram geradas imagens estáticas a partir de aquisições dinâmicas em modo lista nos seguintes intervalos: 0-60 min, 5-20min, 5-30min, 10-60min, 30-60min e 40-60min. Para quantificação das imagens foi utilizado o método da razão entre a média do SUV (Standard Uptake Value) nas regiões justacortical e periventricular e na substância branca, denominado SUVRWM. Os resultados mostram grande variação do SUVRWM nos intervalos que incluem o período de perfusão do radiotraçador. Existe uma maior estabilidade do SUVR ${ }^{\mathrm{WM}}$ nos últimos intervalos avaliados (30-60min e 40-60min), tanto para o grupo controle, como para os pacientes com EM. Conclui-se que o melhor intervalo para aquisição da imagem PET estática para quantificação é de 40 a 60 minutos após a administração, o que significa uma imagem adquirida 40 min após a injeção do radiotraçador $\left[{ }^{11} \mathrm{C}\right]-(R)$-PK11195, por um período de $20 \mathrm{~min}$.
\end{abstract}

Palavras-chave: $\left[{ }^{11} \mathrm{C}\right]-(\mathrm{R})-\mathrm{PK} 11195$; esclerose múltipla; PET; intervalo temporal; quantificação.

\begin{abstract}
$\left[{ }^{11} \mathrm{C}\right]-(R)-P K 11195$ radiotracer shows microglia affinity in PET images and can be used as neuroinflammatory disease indicator, such as in Multiple Sclerosis (MS). There is no consensus about appropriate time interval to generate static PET images with $\left[{ }^{11} \mathrm{C}\right]-(R)-P K 11195$. The aim of this study is to define the most appropriate time interval to generate static brain PET images with [11 C]-(R)-PK11195 for quantification. For this study, images from 10 remitent-recurrent MS patients and 5 healthy controls were used. Static images were generated from list-mode dynamic acquisition in the following time intervals: 0 60min, 5-20min, 5-30min, 10-60 min, 30-60min e 40-60min. The ratio between SUV mean of juxtacortical and periventricular regions and normal appearing white matter, denominated SUVRWM, was used for image quantification. Results shown high variation in time intervals that include radiotracer perfusion. SUVRWM higher stability was observed in two time intervals (30-60min and 40-60min), for both control and MS patients groups. In conclusion, the best acquisition time interval to generate static PET images for quantification is from 40 to 60 minutes after administration, meaning an image acquired 40 minutes after $\left[{ }^{11} \mathrm{C}\right]-(R)-P K 11195$ injection, during $20 \mathrm{~min}$.
\end{abstract}

Keywords: $\left[{ }^{11} \mathrm{C}\right]-(R)-\mathrm{PK} 11195$; multiple sclerosis; PET; time interval; quantification.

\section{Introdução}

As imagens de tomografia por emissão de pósitrons ou PET (Positron Emission Tomography) e as imagens por ressonância magnética ou MRI (Magnetic Resonance Imaging) têm sido amplamente utilizadas no imageamento cerebral de doenças neurodegenerativas ${ }^{1,2}$.

Dentre as doenças neurodegenerativas, a Esclerose Múltipla (EM) possui grande impacto social por afetar adultos jovens, embora seja de baixa prevalência ${ }^{3}$.
A EM é uma doença autoimune, de caráter degenerativo, cuja neuroinflamação progride de um estado de inflamação ativa para a desmielinização e morte axonal ${ }^{1}$. A ativação microglial é um indicador de doença inflamatória cerebral, estando presente na EM.

A inflamação ativa na EM é observada em MRI como uma lesão com hipersinal na imagem ponderada em T2. Em MRI ponderada em T1, a lesão de EM aparece como hipossinal, sendo somente visível quando o processo inflamatório termina com a morte do neurônio ${ }^{5}$. 
Os critérios para o diagnóstico da EM incluem a presença de uma ou mais lesões em duas das quatro regiões consideradas características da doença: justacortical, periventricular, infratentorial e medula espinhal ${ }^{4}$.

Uma escolha importante para o imageamento da ativação microglial in vivo com imagens PET, utiliza o componente PK11195 (1-(2-clorofenil)-Nmetil-N-(1-metilpropil)-3-isoquinolina carboxamida) marcado com ${ }^{11} \mathrm{C}$. Este radiofármaco é um indicador de doença inflamatória cerebral ativa com ampla aplicabilidade ${ }^{6}$. Assim, com a imagem PET, é possível quantificar a captação do [ $\left[{ }^{11} \mathrm{C}\right]-(\mathrm{R})-$ PK11195, relacionando-a com a ativação microglial.

$\mathrm{Na}$ clínica, imagens de PET costumam ser analisadas qualitativamente, mediante a comparação visual entre a captação nos tecidos que possuem alguma patologia e nos tecidos saudáveis.

Uma forma de análise semiquantitativa utiliza o SUV (Standardized Uptake Value), definido como a razão entre a concentração de atividade no tecido $(\mathrm{kBq} / \mathrm{mL})$ e a atividade injetada por unidade de massa do paciente $(\mathrm{kBq} / \mathrm{g})$, corrigida para 0 decaimento radioativo. Entretanto, o SUV é afetado por diversos fatores, tais como a distribuição do radiotraçador, as características antropométricas do paciente, o intervalo de tempo entre a administração do radiofármaco e a aquisição, o método de reconstrução e o tamanho da região de interesse para medição do SUV7.

Uma alternativa para a semiquantificação é o SUVR (Standardized Uptake Value Ratio), dado pela razão entre o SUV de um tecido de interesse e um tecido de referência. O SUVR é adimensional, sendo calculado através da razão entre a concentração de atividade de uma região de interesse e um tecido de referência, em um intervalo temporal pré-fixado.

Os métodos semiquantitativos de imagens PET com [ $\left.{ }^{11} \mathrm{C}\right]-(\mathrm{R})-\mathrm{PK} 11195$ são aplicados em imagens reformatadas a partir de um intervalo temporal definido a partir de uma aquisição dinâmica, usualmente adquirida em modo lista (list-mode). Diversos intervalos temporais já foram avaliados para a semiquantificação com o SUV em imagens PET com [ $\left[{ }^{11} \mathrm{C}\right]-(\mathrm{R})-\mathrm{PK} 11195$, tais como: 5 a 30 min, por ser o intervalo no qual o pseudo-equilíbrio é alcançado em áreas de alta ativação microglial ${ }^{8}$; 40 a 60 min, por ser o período no qual as curvas de atividade ao longo do tempo apresentam declínio similar ao estado estável (40 min após a injeção do radiotraçador) ${ }^{9,10}$. Outros estudos utilizaram intervalos temporais distintos, tais como: 0 a $60 \min ^{11}, 10$ a $60 \min ^{12}$ e 30 a 60 min $^{13}$.

Entretanto, ainda não há um consenso sobre o intervalo temporal ideal para gerar imagens PET estáticas com $\left[{ }^{11} \mathrm{C}\right]-(R)-\mathrm{PK} 11195$ para a semiquantificação na EM utilizando o SUV ou SUVR.

O objetivo deste trabalho é determinar 0 intervalo temporal mais adequado para a geração de imagens PET cerebrais estáticas adquiridas com [ $\left.{ }^{11} \mathrm{C}\right]-(R)-\mathrm{PK} 11195$ para a quantificação das imagens para o diagnóstico da esclerose múltipla.

\section{Materiais e Métodos}

\subsection{Dados do estudo}

As imagens PET/CT cerebrais com $\left[{ }^{11} \mathrm{C}\right]-(R)$ PK11195 e imagens por RM cerebrais foram adquiridas de voluntários do projeto aprovado pelo Comitê de Ética em Pesquisa (CAAE 23949813.7.0000.5336, aprovado em 06/06/2015, sob parecer 1.094.228).

Os voluntários estão divididos em dois grupos: sendo 5 (cinco) sujeitos saudáveis no grupo controle [24 \pm 2 anos] e 10 (dez) pacientes com EM remitente-recorrente [28 \pm 3 anos].

\subsection{Aquisição das imagens}

Ambas as aquisições das imagens (PET/CT e MRI) aconteceram no mesmo dia. A atividade administrada foi de $(560 \pm 100) \mathrm{MBq}$ de $\left[{ }^{11} \mathrm{C}\right]-(\mathrm{R})$ PK11195 para aquisição PET/CT. O radiotraçador foi produzido pelo laboratório do Centro de Produção de Radiofármacos do Instituto do Cérebro do Rio Grande do Sul, na PUCRS.

As imagens PET/CT foram adquiridas no modo lista, em um equipamento GE Discovery 600 (General Electric Healthcare), com cristais detectores tipo óxido de germânio bismuto (BGO), ao longo de $60 \mathrm{~min}$, desde o momento da administração em bolus do [ $\left.{ }^{11} \mathrm{C}\right]-(\mathrm{R})-\mathrm{PK} 11195$.

As imagens PET/CT foram reconstruídas com $300 \mathrm{~mm}$ de campo de visão, utilizando o algoritmo iterativo de reconstrução VUE Point HD, frequência de corte do filtro de suavização de $4,0 \mathrm{~mm}, 32$ subconjuntos e duas iterações. A matriz da imagem possui $192 \times 192$ pixels, 16-bits por pixel (resolução de 0,640 pixels $/ \mathrm{mm}$ ), tamanho de pixel de $1,56 \times 1,56 \mathrm{~mm}^{2}$ e 47 cortes axiais de $3,27 \mathrm{~mm}$ de espessura cada. A atenuação foi corrigida utilizando a tomografia computadorizada por raios $X$ adquirida anteriormente ao início da aquisição da imagem PET. Demais correções necessárias para quantificação (normalização, decaimento, espalhamento e coincidências aleatórias) também foram aplicadas.

As imagens por MR foram adquiridas em um equipamento de ressonância magnética GE Healthcare Signa HDxt de 3,0 T (General Electric Healthcare). Foram adquiridas imagens estruturais de alta resolução ponderadas em T1, utilizando sequência BRAVO ${ }^{\mathrm{TM}}$, com TR de 2400 ms; TE de $16 \mathrm{~ms}$; campo de visão de $220 \mathrm{~mm}$; voxels isotrópicos de $1 \mathrm{~mm}$. As imagens ponderadas em T1 apresentam matriz de $512 \times 512 \times 196$ pixels $\left(240,03 \times 240,03 \times 196,00 \mathrm{~mm}^{3}\right), 16$-bits por pixel, resolução de 2,133 pixels $/ \mathrm{mm}$, tamanho de voxel de $0,47 \times 0,47 \times 1,00 \mathrm{~mm}^{3}$, e intervalo entre quadros de $6,12 \mathrm{~ms}$. 


\subsection{Reformatação das imagens $P E T / C T$}

Imagens PET estáticas foram geradas a partir da média de alguns dos intervalos temporais descritos na literatura, conforme apresentado anteriormente, utilizando a aquisição dinâmica em modo lista:

(A) 0 a $60 \mathrm{~min}^{11}$ (toda aquisição);

(B) 5 a $20 \mathrm{~min}^{3}$ (15 min, após perfusão);

(C) 5 a $30 \min ^{8}$ (25 min, após perfusão);

(D) 10 a 60 min $^{12}$ (últimos $50 \mathrm{~min}$ );

(E) 30 a $60 \min ^{13}$ (últimos $30 \mathrm{~min}$ ); e

(F) 40 a 60 min $^{9,10}$ (últimos 20 minutos).

\subsection{Quantificação das imagens}

Para quantificação das imagens foi utilizado o método semiquantitativo SUVRWM, definido como a razão entre a concentração de atividade nas regiões justacortical e periventricular (JPV) e a concentração de atividade na substância branca aparentemente saudável ou WM (White Matter).

Para extração dos dados das regiões justacortical e periventricular foram utilizadas as máscaras segmentadas a partir das sobreposições das regiões de substância cinzenta e substância branca, utilizando as imagens por MR, com o aplicativo Statistical Parametric Mapping, do MATLAB, conforme descrito em trabalho anterior ${ }^{14}$.

\subsection{Análise estatística}

Para determinar o melhor intervalo temporal foi analisada a variabilidade estatística dos dados através do desvio padrão da média do SUVRWM nas regiões JPV, para cada um dos seis intervalos temporais apresentados na seção 2.3. O intervalo temporal ideal para a quantificação de imagens estáticas de PET deve possuir menor desvio padrão para ambos os grupos estudados (controles e pacientes com EM). Para comparar as variabilidades, foi calculado o coeficiente de variação (COV), definido pela razão entre o desvio padrão e a média, multiplicado por $100 \%$.

Para avaliar se os intervalos temporais apresentam diferença estatisticamente significativa entre os SUVRWM dos pacientes com EM e grupo controle, foi utilizado o teste t de Student $(p=0,05)$.

\section{Resultados}

A Figura 1 apresenta as médias de SUV nas regiões JPV e respectivo desvio padrão para os pacientes com EM e grupo controle, ao longo de toda a aquisição dinâmica. Os gráficos de SUV ao longo do tempo são denominados curvas de atividade-tempo ou TACs (Time Activity Curves).

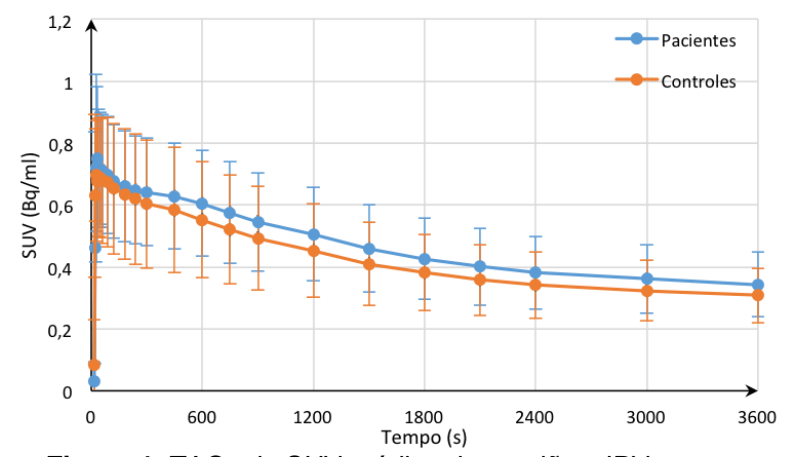

Figura 1. TACs do SUV médios das regiões JPV para os grupos de pacientes com EM e grupo controle.

As TACs das médias de SUV da WM aparentemente normal dos pacientes com EM e grupo controle são apresentadas na Figura 2.

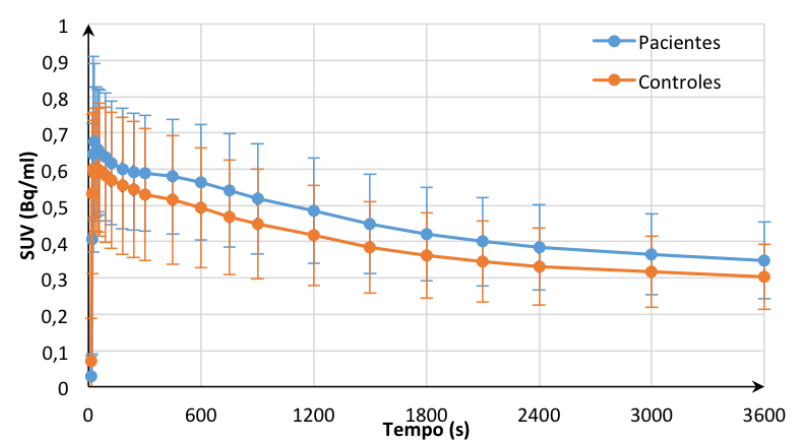

Figura 2. TACs das médias de SUV da WM para os grupos de pacientes com EM e grupo controle.

A partir dos SUVs, foram calculados, para os pacientes com EM e grupo controle, os SUVRWM para as regiões JPV, nos intervalos temporais estudados. Os resultados das médias e respectivos desvios padrão, os valores de COV e a significância do teste $t$ de Student, para cada intervalo temporal investigado, são apresentados na Tabela 1.

Tabela 1. Média e desvio padrão do SUVR ${ }^{\mathrm{WM}}$, COV e nível de significância do teste $t$ de Student, em cada intervalo temporal, para os grupos controle e pacientes com EM.

\begin{tabular}{cccccc}
\hline Intervalo & $\begin{array}{c}\text { SUVR }^{\text {WM }} \\
\text { Pacientes }\end{array}$ & COV & $\begin{array}{c}\text { SUVR } \\
\text { Controles }\end{array}$ & COV Valor p \\
\hline 0 - 60min & $1,06 \pm 0,06$ & $5,7 \%$ & $1,11 \pm 0,05$ & $4,5 \%$ & 0,02 \\
5 - 20min & $1,07 \pm 0,04$ & $3,7 \%$ & $1,11 \pm 0,02$ & $1,8 \%$ & $<0,01$ \\
$5-30 \mathrm{~min}$ & $1,06 \pm 0,04$ & $3,8 \%$ & $1,10 \pm 0,03$ & $2,7 \%$ & $<0,01$ \\
$10-60 \mathrm{~min}$ & $1,02 \pm 0,04$ & $3,9 \%$ & $1,06 \pm 0,04$ & $3,7 \%$ & $<0,01$ \\
$30-60 \mathrm{~min}$ & $1,00 \pm 0,03$ & $3,0 \%$ & $1,03 \pm 0,02$ & $1,9 \%$ & 0,02 \\
$40-60 \mathrm{~min}$ & $0,99 \pm 0,02$ & $2,0 \%$ & $1,02 \pm 0,02$ & $2,0 \%$ & 0,03 \\
\hline
\end{tabular}

A Figura 2 apresenta o gráfico do desvio padrão da média do SUVRWM em cada intervalo temporal investigado, para os dados do grupo controle e dos pacientes com EM. 


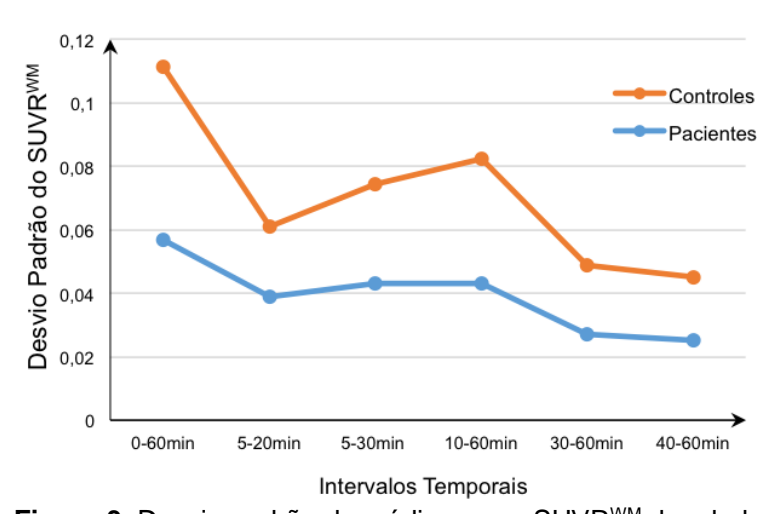

Figura 3. Desvio padrão da média para o SUVR ${ }^{\mathrm{WM}}$ dos dados do grupo controle e dos pacientes com EM em cada intervalo temporal investigado.

\section{Discussão}

Observa-se, através das TACs (Figuras 1 e 2), que a captação do radiofármaco $\left[{ }^{11} \mathrm{C}\right]-(R)-\mathrm{PK} 11195$ é sistematicamente maior para os pacientes com EM em relação ao grupo controle, tanto para as regiões justacortical e periventricular, como para a substância branca aparentemente normal. Esta maior captação do $\left[{ }^{11} \mathrm{C}\right]-(R)-\mathrm{PK} 11195$ pelos pacientes com EM é consistente com a hipótese de ativação microglial devido à neuroinflamação.

Os SUVs nas regiões de interesse (regiões JPV e WM) avaliadas neste trabalho apresentam concordância com resultados anteriores obtidos por Kumar e colaboradores $(2012)^{3}$, que utilizam o intervalo temporal de 5 a 20 min.

O teste t de Student mostra que os SUVRWM nas regiões JPV são estatisticamente diferentes para os pacientes com EM e grupo controle, em todos os intervalos temporais investigados $(p<0,05)$.

Não foram encontradas pesquisas que avaliam 0 uso do SUVRWM nas regiões JPV utilizando imagens PET com $\left[{ }^{11} \mathrm{C}\right]-(R)-\mathrm{PK} 11195$ para 0 diagnóstico de esclerose múltipla.

Os desvios padrão das médias de SUVR'WM (Figura 3) mostram maior variação nos intervalos que incluem o período de perfusão do radiotraçador (primeiros $10 \mathrm{~min}$ após a administração), particularmente para os pacientes com EM. Assim, a inclusão do período de perfusão no intervalo temporal não é adequado para a produção da imagem PET estática.

A maior estabilidade de SUVRWM ocorre nos dois últimos intervalos avaliados (30-60min e 40$60 \mathrm{~min}$ ), evidenciado pelos menores valores de desvio padrão da média na Figura 3 e menores COV (Tabela 1), tanto para o grupo controle, como para os dados provenientes dos pacientes com EM.

\section{Conclusões}

Conclui-se que o melhor intervalo para aquisição da imagem PET estática para quantificação do radiotraçador [11C]-(R)-PK11195 é de 40 a 60 minutos após a administração, o que significa uma imagem de $20 \mathrm{~min}$, adquirida $40 \mathrm{~min}$ após a injeção. Este intervalo produz imagens cujo SUVRWM nas regiões justacortical e periventricular possuem a menor variabilidade, para a população estudada, permitindo a diferenciação entre pacientes com esclerose múltipla e controles saudáveis.

Maiores estudos são necessários para avaliar este resultado com maior número de pacientes, assim como correlacionar os SUVWM com a progressão da esclerose múltipla.

\section{Referências}

1. Banati, R. B. et al. The peripheral benzodiazepine binding site in the brain in multiple sclerosis: quantitative in vivo imaging of microglia as a measure of disease activity. Brain a J. Neurol. 123, 2321-37 (2000).

2. Chauveau, F., Boutin, H., Van Camp, N., Dollé, F. \& Tavitian, B. Nuclear imaging of neuroinflammation: A comprehensive review of $\left[{ }^{11} \mathrm{C}\right] \mathrm{PK} 11195$ challengers. Eur. J. Nucl. Med. Mol. Imaging 35, 2304-2319 (2008).

3. Kumar, A. et al. Evaluation of age-related changes in translocator protein (TSPO) in human brain using ${ }^{11} \mathrm{C}-[\mathrm{R}]-\mathrm{PK} 11195$ PET. J Neuroinflammation 9, 232 (2012).

4. Polman, C. H. et al. Diagnostic criteria for multiple sclerosis: 2010 revisions to the McDonald criteria. Ann. Neurol. 69, 292-302 (2011).

5. Giannetti, P. et al. Microglia activation in multiple sclerosis black holes predicts outcome in progressive patients: An in vivo $\left[{ }^{11} \mathrm{C}\right](\mathrm{R})-\mathrm{PK} 11195-\mathrm{PET}$ pilot study. Neurobiol. Dis. 65, 203-210 (2014).

6. Lammertsma, A. A. \& Hume, S. P. Simplified reference tissue model for PET receptor studies. Neuroimage 4, 153-8 (1996).

7. Boellaard, R. Standards for PET Image Acquisition and Quantitative Data Analysis. J. Nucl. Med. 50, 11S-20S (2009).

8. Anderson, A. N. et al. A systematic comparison of kinetic modelling methods generating parametric maps for $\left[{ }^{11} \mathrm{C}\right]-(\mathrm{R})$ PK11195. Neuroimage 36, 28-37 (2007).

9. Debruyne, J. Semiquantification of the peripheral-type benzodiazepine ligand $\left[{ }^{11} \mathrm{C}\right]$ PK11195 in normal human brain and application in multiple sclerosis patients. Acta Neurol. Belg. 102, 127-135 (2002).

10. Versijpt, J. et al. Microglial imaging with positron emission tomography and atrophy measurements with magnetic resonance imaging in multiple sclerosis: a correlative study. Mult. Scler. 11, 127-134 (2005).

11. Politis, M. et al. Increased PK11195 PET binding in the cortex of patients with MS correlates with disability. Neurology 79, 52330 (2012).

12. Hammoud, D. a et al. Imaging glial cell activation with $\left[{ }^{11} \mathrm{C}\right]-\mathrm{R}-\mathrm{PK} 11195$ in patients with AIDS. J. Neurovirol. 11, 346-355 (2005).

13. Schuitemaker, A. et al. SPM analysis of parametric (R)-[11 C]PK11195 binding images: Plasma input versus reference tissue 
parametric methods. Neuroimage 35, 14731479 (2007).

14. Narciso, L. D. L. et al. Semiquantification Study of [ $\left.{ }^{11} \mathrm{C}\right]-(\mathrm{R})-\mathrm{PK} 11195$ PET Brain Images in Multiple Sclerosis. Rev. Bras. Física Médica 10, (2016).

\section{Contato:}

Phelipi Nunes Schuck

Av. Ipiranga, 6681 - Partenon, Porto Alegre, RS.

E-mail: phelipi.schuck@acad.pucrs.br 